\title{
The effect of stress on the cross-slip energy in face-centered cubic metals: a study using dislocation dynamics simulations and line tension models
}

\author{
M. Longsworth, M. Fivel \\ Univ. Grenoble Alpes, CNRS, Grenoble INP, SIMaP, Grenoble 38000, France
}

\begin{abstract}
Dislocation dynamics simulations were used to calculate the energy barrier of cross-slip via FriedelEscaig mechanism in face centered-cubic copper. The energy barrier in the unstressed case was found to be $1.9 \mathrm{eV}$, as reported by B. Ramírez et al. ${ }^{[1]}$. The energy barrier was reduced by applying an external stress. The most effective way of reducing it, was by applying a compressive stress on the glide plane. Furthermore, it was confirmed using dislocation dynamics simulations, that both the Schmid and Escaig stress have a comparable effect in reducing the energy barrier, in qualitative agreement with the atomistic simulations performed by K. Kang et al. ${ }^{[2]}$ in face-centered cubic nickel. Most of the energy barrier values for stressed cross-slip fell within the experimental error of $1.15 \pm 0.37 \mathrm{eV}$ measured by J. Bonneville et al. ${ }^{[3]}$. Moreover, the activation enthalpy obtained from K. Kang's et al. ${ }^{[2]}$ line tension model of cross-slip and the general expression for the activation enthalpy proposed by A. Malka-Markovitz et al. ${ }^{[4]}$ were in good quantitative agreement with the simulation results. Hence, both could be used to calculate the activation enthalpy of screw segments in dislocation dynamics simulations.
\end{abstract}

Keywords:

activation energy, cross-slip, dislocation dynamics, line tension model, Escaig stress, Schmid stress

\section{Introduction}

Cross-slip is a thermally-activated process by which screw dislocations change their glide plane, allowing them to overcome obstacles and populate other slip planes ${ }^{[5]}$. It plays an important role in two competing processes during plastic deformation ${ }^{[6]}$. On the one hand, it causes dynamic recovery during stage III by dislocation annihilation ${ }^{[7]}$, and other, it contributes to work hardening by dislocation multiplication ${ }^{[8,9]}$.

Since cross-slip is a thermally activated process, its rate is controlled by an Arrhenius-like equation. Many dislocation dynamics (DD) simulations have successfully model plastic deformation in crystals ${ }^{[10-14]}$. Most of them use the rate equation proposed by L. Kubin et al. ${ }^{[15]}$. In their model, the cross-slip probability explicitly depends on the resolved shear stress at the onset of stage-III 
plastic deformation, which is a macroscopic parameter obtained from the stress-strain curve of the material.

This probability law has demonstrated to be robust enough to allow the formation of persistent slip bands in the low-cycle fatigue regime ${ }^{[16]}$, or to study the recovery dynamics in copper ${ }^{[17]}$. However, it is not sufficient in many situations. As an example, in the case of irradiated materials, the local level of stress is very high because of the large number of obstacles associated with the defect density. In this case, the cross-slip events are too frequent ${ }^{[18]}$. Contrarily, when the stress level is very low, the probability of inducing cross-slip events will be very low or nonexistent.

Based on the work of L. Kubin et al. ${ }^{[15]}$, A. Hussein et al. ${ }^{[19]}$ recently proposed a more physical rate equation that depends only on microstructure parameters, e.g. the energy barrier required to form a constriction point on a screw dislocation, the Debye frequency of the material, the activation volume and the local stress. This allowed them to implement DD simulations considering the different cross-slip mechanisms identified from molecular dynamics (MD) simulations. Quantifying the effect of stress on the activation barrier is thus of great importance to generate realistic DD simulations.

The two most plausible cross-slip models for Face-Centered Cubic (FCC) crystals are the Fleischer (FL) and Friedel-Escaig (FE) mechanisms.

The FL model was developed by R. Fleischer ${ }^{[20]}$. In his model, the leading partial in a stackingfault dissociates into two partials; one glides on the cross-slip plane and the other remains sessile at the intersection of the two planes (stair-rod dislocation). The trailing partial then reacts with the stair-rod dislocation to form another Shockley partial in the conjugate plane, which completes the cross-slip process.

The FE model was developed by B. Escaig ${ }^{[21]}$ based on J. Friedel's ideas ${ }^{[22]}$. Moreover, he used the line tension (LT) model of cross-slip developed by A. Stroh ${ }^{[23]}$ to study the effect of the widening stress (Escaig stress) on the energy barrier. In the FE model, the extended dislocation stops moving in the glide plane due to an obstacle. An already-existing constriction then splits into two halves and then separate in the cross-slip plane. This process proceeds spontaneously due to the stress on the cross-slip plane until the dissociation is completed.

Although there have been some controversy on which cross-slip model prevails ${ }^{[24]}$, recent atomistic simulations confirm that both FL and FE mechanisms can occur. The determination of which process would dominate depends on many factors.

C. Jin et al. ${ }^{[25]}$ used the nudged-elastic-band method to study FCC Al. They concluded that there is a critical dislocation length below which the screw dislocation cross-slips only via FL mechanism, whereas longer ones would cross-slip either via FL or FE mechanisms, depending on the initial and final positions of the dislocation. The critical length they found was of the order of $22 b$, where $b$ is the magnitude of the Burgers vector.

$\mathrm{S} . \mathrm{Xu}$ et al. ${ }^{[26]}$ reported similar findings in FCC Ni using dynamic concurrent atomistic-continuum and MD simulations. They found that screw dislocations of length $6.47 \mathrm{~nm}$ cross-slip via FL mechanism, whereas longer dislocations (of length $12.94 \mathrm{~nm}$ ) can cross-slip via either the FE or FL mechanisms. They also suggested that the critical shear stress for both FE and FL mechanisms 
depends on the dislocation length.

Recently, W. Kuykendall et al. ${ }^{[27]}$ used atomistic simulations to examine the effect of stress on the energy barrier of cross-slip via FE and FL mechanisms in FCC Ni. They concluded that the FE mechanism prevails when the Escaig stress on the glide plane is dominant, and that increasing the Schmid and Escaig stress on the cross-slip plane promotes the FL mechanism.

The material of study in this work is FCC $\mathrm{Cu}$ due to its broad literature. The mechanism of cross-slip in $\mathrm{Cu}$ has been fully examined using atomistic simulations and it was reported that dislocations in this material cross-slip via $\mathrm{FE}$ mechanism ${ }^{[28,29]}$. The activation energy of $\mathrm{Cu}$ has been calculated using both elasticity theory and atomistic simulations. However, the vast majority of energy barrier calculations employ atomistic simulations ${ }^{[29-34]}$.

Alternatively, B. Ramírez et al. ${ }^{[1]}$ used a network-based formulation of dislocation dynamics to study the effect of a general stress on the energy barrier of cross-slip via FE mechanism. They found that the stress-free activation energy in $\mathrm{Cu}$ is $1.9 \mathrm{eV}$ when the core is represented by two partials, and that it converges to $1.43 \mathrm{eV}$ when the core is distributed by 20 Volterra partial fractional dislocations, the later value being in better agreement with the experimental results. They also found that the cross-slip energy reduces to $0.62 \mathrm{eV}$ in presence of a Lomer-Cottrel junction.

In recent years, K. Kang et al. ${ }^{[2]}$ extended Escaig's LT model to include the effect of gliding stress (Schmid stress) on the cross-slip plane. Contrary to Escaig's claim that the Schmid stress had a negligible effect on the energy barrier, they found that it had a comparable effect to the Escaig stress. They calculated the energy barrier in FCC Ni using atomistic methods at different stress conditions and found good qualitative agreement with their LT model.

More recently, A. Malka-Markovitz et. al. ${ }^{[4]}$ solved Strohs's LT model of cross-slip ${ }^{[23]}$ exactly by linearizing the interaction force between the partials. They obtained a general expression for the activation enthalpy as function of the elastic constants and local stress.

The DD simulations of fatigue mostly rely on the energy barrier, which is often calculated using atomistic simulations. However, the interatomic potential quantitatively affects the simulation results $^{[30]}$. Hence, they may not be reliable. On the other hand, in DD simulations, the core-width used to compute the elastic energy can be determined using the theory developed by G. Schöck ${ }^{[35]}$, as suggested by B. Ramírez et al. ${ }^{[1]}$. Moreover, DD simulations naturally enable the possibility to study the effect of complex structures on the energy barrier. In this way, they offer an attractive alternative to large-scale atomic simulations.

The contribution of this work is twofold. First, the effect of stress on the cross-slip activation enthalpy is carefully calculated using DD simulations, which is an interesting alternative to the common atomistic approach found in the literature. Second, the results are compared with those obtained from two recent LT models of cross-slip in order to verify their consistency. The objective is to determine whether the aforementioned LT models could be reliably used in DD simulations to calculate the activation enthalpy of screw segments.

The rest of this paper is organized as follows. Section 2 introduces the DD simulation methodology, K. Kang's et al. ${ }^{[2]}$ LT model of cross-slip and the general expression for the activation enthalpy proposed by A. Malka-Markovitz et. al. ${ }^{[4]}$. Section 3 describes the effect of stress on the activation 
enthalpy obtained using DD simulations and the two LT models. Finally, Section 4 presents the summary and conclusions of this work.

\section{Methodology}

\subsection{DD simulations}

\subsubsection{Force computation}

Dislocation dynamics is a modeling approach to study crystal plasticity, in which dislocation lines are discretized in linear segments and their motion is determined ${ }^{[36]}$. A segment is defined by two nodes on its ends and a tangent line $\hat{\xi}$ that connects them. The Burgers vector $\mathbf{b}$ associated to it indicates the displacement's magnitude and direction as the segment moves.

The force exerted by the local stress at each node is calculated using the Peach-Köhler formula. Similarly to other discrete DD codes ${ }^{[37]}$, NuMoDis ${ }^{[38]}$ uses the formalism developed by A. Arsenlis et al. ${ }^{[39]}$ to compute the nodal forces. This force affects the nodal velocity through a given mobility law. Thus, the dislocation dynamics can be determined by updating the position of all nodes after a simulation step.

In order to study cross-slip in FCC crystals, the dissociated character of dislocations must be considered. The SF produces a force that tends to attract the partials towards each other, compensating the elastic repulsion between them. This results in an equilibrium separation distance in the SF ribbon.

Perfect dislocations dissociate into partials if it decreases its elastic energy. However, discrete DD codes are based on nodal forces and do not consider the systems energetics to determine its evolution. In consequence, E. Martinez et al. ${ }^{[40]}$ proposed to account for the intrinsic SF energy $\gamma_{i}$ by introducing a SF force $\left(\mathbf{f}_{s f}\right)$. They derived that $\mathbf{f}_{s f}$ should be of magnitude $\gamma_{i}$, and perpendicular to both the tangent line and SF plane $\hat{\mathbf{n}}$. The equation satisfying these conditions is given by:

$$
\mathbf{f}_{s f}=\gamma_{i} \hat{\mathbf{n}} \times \hat{\xi}
$$

In NuMoDis ${ }^{[38]}$, the SF ribbon is defined by an ordered list of nodes conforming the dislocation partials and the plane that contains them. The SF forces acting on a partial, in order to be physical, must point towards the opposite partial. Thus, given a slip plane orientation, the node order defining the circuit must be such that the SF force given by Eq. 1 satisfy this condition.

\subsubsection{Velocity computation}

In FCC metals, the relationship between forces and velocities is linear due to the small intrinsic lattice resistance ${ }^{[41]}$. The total force at a node $\mathbf{f}$ is given by $\mathbf{f}=\mathbf{f}_{P K}+\mathbf{f}_{s f}$, where $\mathbf{f}_{P K}$ is the Peach-Köhler force. The projections of $\mathbf{f}_{P K}$ and $\mathbf{f}_{s f}$ on the glide plane act perpendicular to the line segment. Therefore, the mobility law is of the form:

$$
\mathbf{f}=\underline{\underline{B}} \cdot \mathbf{v}
$$


where $\mathbf{v}$ is the nodal velocity and $\underline{\underline{B}}$ the viscosity matrix:

$$
\underline{\underline{B}}(\hat{\xi})=B(\mathbf{I}-\hat{\xi} \otimes \hat{\xi})
$$

where $B$ is the viscosity coefficient.

Thus, the nodal velocity is obtained by inverting the viscosity matrix from Eq. 2:

$$
\mathbf{v}=\underline{\underline{B}}^{-1} \cdot \mathbf{f}
$$

At each simulation step, the force at every node due to the influence of all other segments and $\mathrm{SF}$ is computed; the nodal velocities are thus calculated and their new positions are updated.

\subsubsection{Elastic energy}

W. Cai et al. ${ }^{[42]}$ showed that the non-singular interaction energy $E^{n s}$ between a segment with ends $\mathbf{x}_{\mathbf{1}}$ and $\mathbf{x}_{\mathbf{2}}$ and Burgers vector $\mathbf{b}$ with another segment with ends $\mathbf{x}_{\mathbf{3}}$ and $\mathbf{x}_{\mathbf{4}}$ and Burgers vector $\mathbf{b}^{\prime}$ is given by:

$$
\begin{aligned}
& E^{n s}=-\frac{\mu}{8 \pi} \int_{\mathbf{x}_{3}}^{\mathbf{x}_{4}} \int_{\mathbf{x}_{1}}^{\mathbf{x}_{2}} \partial_{k} \partial_{k} R_{a} b_{i} b_{j}^{\prime} d x_{i} d x_{j}^{\prime} \\
& -\frac{\mu}{4 \pi(1-\nu)} \int_{\mathbf{x}_{3}}^{\mathbf{x}_{4}} \int_{\mathbf{x}_{1}}^{\mathbf{x}_{2}} \partial_{i} \partial_{j} R_{a} b_{i} b_{j}^{\prime} d x_{k} d x_{k}^{\prime} \\
& +\frac{\mu}{4 \pi(1-\nu)} \int_{\mathbf{x}_{3}}^{\mathbf{x}_{4}} \int_{\mathbf{x}_{1}}^{\mathbf{x}_{2}} \partial_{k} \partial_{k} R_{a} b_{i} b_{i}^{\prime} d x_{j} d x_{j}^{\prime} \\
& -\frac{\mu \nu}{4 \pi(1-\nu)} \int_{\mathbf{x}_{3}}^{\mathbf{x}_{4}} \int_{\mathbf{x}_{1}}^{\mathbf{x}_{2}} \partial_{k} \partial_{k} R_{a} b_{i} b_{j}^{\prime} d x_{j} d x_{i}^{\prime}
\end{aligned}
$$

where $\mu$ is the shear modulus, $\nu$ the Poisson's ratio, $R_{a}=\sqrt{\mathbf{R} \cdot \mathbf{R}+a^{2}}, \mathbf{R}=\mathbf{x}-\mathbf{x}^{\prime}$, and $a$ the core-width parameter.

The analytical expressions resulting from the solution of Eq. 5 for parallel and non-parallel segments were provided by W. Cai et al. ${ }^{[42]}$. These formulas were used to calculate the interaction and self-energies of the segments in NuMoDis ${ }^{[38]}$.

The core-width parameter $a$ affects the elastic energy both qualitatively and quantitatively. G. Schöck ${ }^{[35]}$ developed a theory based on the Peierls framework ${ }^{[43]}$ to obtain a physical value of $a$. He showed that the total energy of a single straight dislocation is given by ${ }^{[35]}$ :

$$
E^{P}=E_{L}(\phi)\left[\ln \left(\frac{r}{2 \bar{w}(\phi)}\right)+1\right]
$$

where $\phi$ is the angle between the Burgers vector and the line direction, $r$ is the outer cut-off radius, $\bar{w}(\phi)$ is the average width of its edge and screw components, and $E_{L}(\phi)$ is the pre-logarithmic elastic line energy factor: 


$$
E_{L}(\phi)=\frac{\mu b^{2}}{4 \pi}\left(\cos ^{2}(\phi)+\frac{\sin ^{2}(\phi)}{1-\nu}\right)
$$

G. Schöck ${ }^{[5]}$ argued that $\bar{w}(\phi)$ can be approximated as the width of a single dislocation in an isotropic medium passing through two equilibrium positions i.e. with SF energy $\gamma=\gamma_{u}{ }^{[35]}$ :

$$
\frac{\bar{w}(\phi)}{b}=\frac{\cos ^{2}(\phi)+\sin ^{2}(\phi) /(1-\nu)}{b / c+2 \pi^{2} \gamma_{u} /(\mu b)}
$$

where $c$ is the interplanar glide plane spacing, $\gamma_{u}$ the unstable SF energy and $b$ is the magnitude of the Burgers vector.

Using W. Cai's et al. ${ }^{[42]}$ model, the line energy for screw $E_{S}$ and edge dislocation $E_{E}$ are found to be ${ }^{[35]}$ :

$$
\begin{gathered}
E_{S}=\frac{\mu b^{2}}{4 \pi} \ln \left(\frac{r \sqrt{e}}{a_{S}}\right) \\
E_{E}=\frac{\mu b^{2}}{4 \pi(1-\nu)} \ln \left(\frac{r}{a_{E}}\right)
\end{gathered}
$$

where $a_{S}$ and $a_{E}$ are the core spreading parameters of the screw and edge components, respectively, and $e$ is the base of the natural logarithm.

The continuum description in Eqs. 9 and 10 must be consistent with the Peierl's atomistic treatment in eq. 6. Imposing this condition, $a_{S}$ and $a_{E}$ are required to be ${ }^{[35]}$ :

$$
a_{S}=\frac{2 \bar{w}(0)}{\sqrt{e}} \quad \text { and } \quad a_{E}=\frac{2 \bar{w}(\pi / 2)}{e}
$$

Following the work of B. Ramírez et al. ${ }^{[1]}$, the core-width parameter can be considered as an averaged value of $a_{S}$ and $a_{E}$ :

$$
a \approx \frac{2 \bar{w}(0)}{e}=\frac{2}{e}\left(\frac{b}{b / c+2 \pi^{2} \gamma_{u} /(\mu b)}\right)
$$

\subsubsection{Effective stacking-fault energy}

Dislocations in FCC crystals dissociate into partials. The dislocation moves due to the action of an external stress. The partial at the front respect to the direction of motion is called the "leading" partial, and the other "trailing" partial. Let $L=\left\{\vec{P}_{1}, \vec{P}_{2}, \ldots, \vec{P}_{N}\right\}$ and $\tilde{L}=\left\{\vec{Q}_{1}, \vec{Q}_{2}, \ldots, \vec{Q}_{N}\right\}$ represent the set of nodes conforming the leading and trailing partials, respectively (see Fig. 1). 


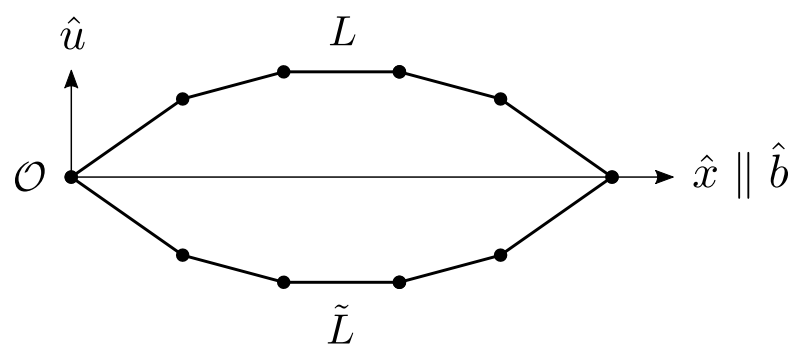

Figure 1: The solid dots denote the nodal positions; the shape of the leading and trailing partials are described by $L$ and $\tilde{L}$, respectively.

The effective SF energy is given by the SF energy $W_{S F}$ plus the work done by the applied forces $W_{a p p}$, which can be calculated as follows:

$$
\begin{gathered}
W_{S F}=\gamma_{i} \sum_{j=1}^{N}\left[\frac{y_{j+1}+y_{j}}{2} \Delta x_{j}-\frac{\tilde{y}_{j+1}+\tilde{y}_{j}}{2} \Delta \tilde{x}_{j}\right] \\
W_{a p p}=\sum_{j=1}^{N} F y_{j} \Delta x_{j}+\tilde{F} \tilde{y}_{j} \Delta \tilde{x}_{j}
\end{gathered}
$$

where:

$y_{j}=\vec{P}_{j} \cdot \hat{u}, \tilde{y}_{j}=\vec{Q}_{j} \cdot \hat{u}, \Delta x_{j}=\left(\vec{P}_{j+1}-\vec{P}_{j}\right) \cdot \hat{b}, \Delta \tilde{x}_{j}=\left(\vec{Q}_{j+1}-\vec{Q}_{j}\right) \cdot \hat{b} \quad$ for $\quad j=1,2, \ldots, N$

$\hat{u}=\hat{n} \times \hat{b}$ is an unitary vector contained in the slip plane of normal $\hat{n}$, perpendicular to the normalized Burgers vector $\hat{b}$. In absence of Schmid stress, the partials are indistinguishable from each other due to the spatial symmetry with respect to the $\hat{x}$ axis. For that reason, the sign of $\hat{u}$ does not affect the energy. However, the leading and trailing partials are univocally defined in the presence of a Schmid stress. In that case, $\hat{u}$ must point towards the bowing-out direction because the energy barrier does not depend on the sign of the Schmid stress ${ }^{[2,4,27]}$.

The proportionality factors $F$ and $\tilde{F}$ are given by:

$$
F=-\frac{b \sigma_{E}}{2 \sqrt{3}}-\frac{b \sigma_{S}}{2} \text { and } \tilde{F}=\frac{b \sigma_{E}}{2 \sqrt{3}}-\frac{b \sigma_{S}}{2}
$$

where the subscripts $E$ and $S$ refer to the Escaig and Schmid stress, respectively.

All the nodes were mobile except those two at the extremes, which simulate the constriction points at the equilibrium configurations. Therefore, the partials always satisfied $y_{1}=\tilde{y}_{1}=y_{N}=\tilde{y}_{N}=0$. 


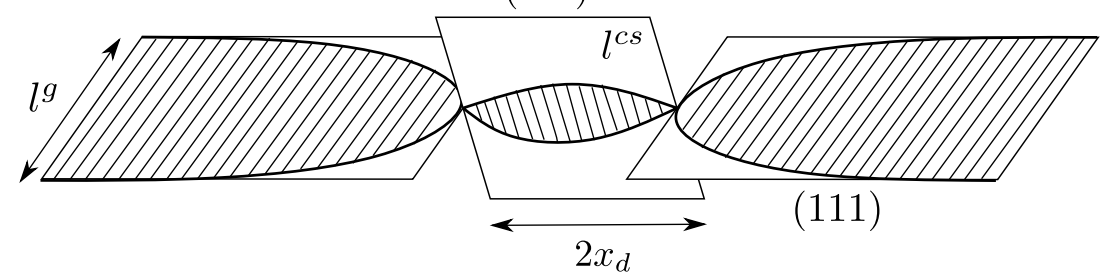

Figure 2: An equilibrium configuration during cross-slip via FE mechanism. The process is completed when the constriction separation tends to infinity. In this limit, the dislocations is completely contained in the conjugate plane with separation between the partials $l^{c s}$ for all $x$ (figure adapted from K. Kang et al. ${ }^{[2]}$ ).

\subsubsection{Cross-slip simulations}

Cross-slip via FE mechanism was assumed in all simulations. The DD code was used to find the equilibrium configurations at different cross-slip stages. The total energy of a configuration consisted of its total elastic energy plus the effective stacking-fault energy, which were computed as described in sections 2.1.3 and 2.1.4. The applied stresses were small enough such that the Frank-Read source remained deactivated in all simulations.

According to the FE mechanism, the dislocation must stop moving before cross-slipping. Therefore, the Schmid stress on the glide plane $\sigma_{S}^{g}$ was always assumed to be zero. The dislocation width in the glide plane was affected by the Escaig stress $\sigma_{E}^{g}$. Soon after stop moving, an alreadyexisting constriction splits into two halves and the dislocation expands in the cross-slip plane until equilibrium is reestablished (see Fig. 2). This occurs in the limit when the distance between the constriction points in the cross-slip plane $2 x_{d}$ tends to infinity.

In DD codes, an infinite dislocation does not really exist. Thus, the initial configuration consisted of a dislocation with length of $1000 \AA$ lying in the $(\overline{111})$ plane. The dislocation was pinned at its ends. The Burgers vector of the partials pointed away from each other as shown in Fig. 3(a). This was a long-enough dislocation such that most part of it attained equilibrium i.e the distance $l^{g}$ between the partials.

An intermediate cross-slip configuration consisted of a semi-infinite dislocation on the glide plane with a constricted cross-slipped segment on the conjugate plane (11) $)$. The Burgers vector of the partials pointed towards each other on the cross-slip plane. The total configuration length was fixed to $1000 \AA$, in agreement with the initial state. The intermediate cross-slip configuration was thus characterized by the distance between its constriction points.

In the limiting case when the constrictions points are infinitely apart, the dislocation must be completely contained in the cross-slip plane (see 3(b)). Unlike in the glide plane, the dislocation was allowed to move by bowing-out on the conjugate plane. The sign of the Schmid stress on the cross-slip plane $\sigma_{S}^{c s}$ was affected only the bowing-out direction, and not the total energy. As in the glide plane, the width of dislocation in the conjugate plane was affected by the Escaig stress $\sigma_{E}^{c s}$. 


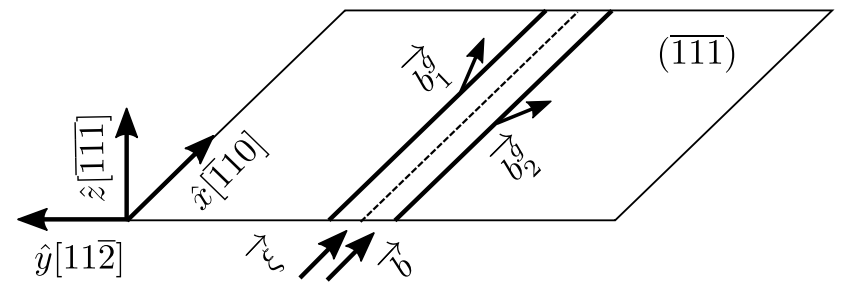

(a)

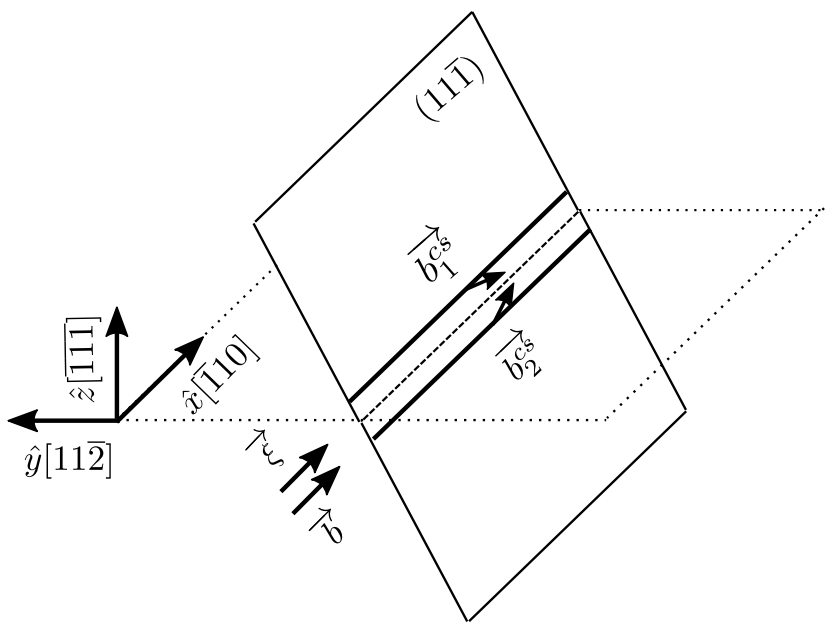

(b)

Figure 3: Simulation setup. (a) The reference configuration consists of a dissociated right-handed screw (RHS) dislocation at equilibrium on the glide plane $(\overline{111})$ with partials' Burgers vector $\overrightarrow{b_{1}^{g}}=$ $\frac{a_{0}}{6}[\overline{1} 2 \overline{1}]$ and $\overrightarrow{b_{2}^{g}}=\frac{a_{0}}{6}[\overline{2} 11]$ pointing away from each other. (b) The final configuration consists of

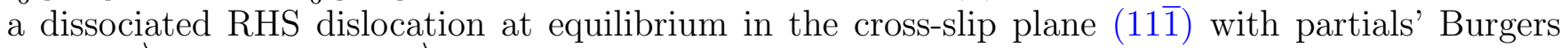
vector $\overrightarrow{b_{1}^{c s}}=\frac{a_{0}}{6}[\overline{1} 21]$ and $\overrightarrow{b_{2}^{c s}}=\frac{a_{0}}{6}[\overline{2} 1 \overline{1}]$ pointing towards each other. 


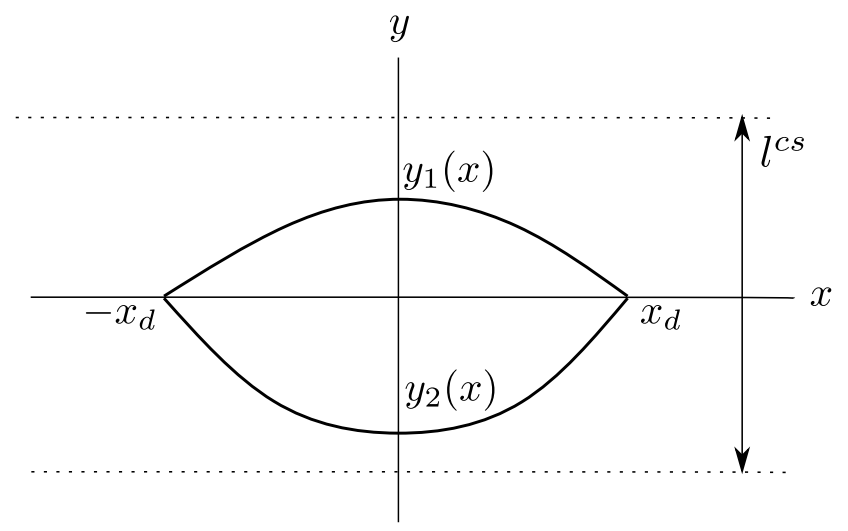

Figure 4: The shape of the partials in the cross-slip plane are described by the $y_{1}(x)$ and $y_{2}(x)$, and satisfy the boundary conditions $y_{1}\left( \pm x_{d}\right)=y_{2}\left( \pm x_{d}\right)=0$ (figure adapted from K. Kang et al. ${ }^{[2]}$ ).

In order to study the effect of $\sigma_{E}^{g}, \sigma_{S}^{c s}$ and $\sigma_{E}^{c s}$, the coordinate system was rotated such that the $[\overline{111}]$ direction matched with the $\hat{z}$ axis and the Burgers vector [110] with the $\hat{x}$ axis. Thus, the stress on the glide and cross-slip planes as function of the tensor components in the Cartesian coordinate system were given by:

$$
\begin{gathered}
\sigma_{S}^{g}=\sigma_{x z} \\
\sigma_{E}^{g}=\sigma_{y z} \\
\sigma_{S}^{c s}=\frac{2 \sqrt{2} \sigma_{x y}-\sigma_{x z}}{3} \\
\sigma_{E}^{c s}=\frac{7 \sigma_{z y}+2 \sqrt{2}\left(\sigma_{y y}-\sigma_{z z}\right)}{9}
\end{gathered}
$$

The energy of an intermediate cross-slip configuration was measured with respect to the energy of the initial state i.e. the energy of the dislocation resting at equilibrium in the glide plane under stress $\sigma_{E}^{g}$. Thus, the energy barrier was defined as the maximum energy among all intermediate cross-slip configurations as function of the distance between the constriction points.

\subsection{Line tension model}

In K. Kang's et al. ${ }^{[2]}$ LT model of cross-slip, the FE mechanism is assumed. Thus, the dislocation does not move before cross-slipping i.e. the Schmid stress on the glide plane is zero $\left(\sigma_{S}^{g}=0\right)$. However, the Escaig stress on the glide plane $\sigma_{E}^{g}$ is not necessarily zero. The equilibrium separation between the partials in the glide plane $l^{g}$ depends on $\sigma_{E}^{g}$ : 


$$
l^{g}=\frac{A}{F^{g}}
$$

where for a screw dislocation dissociated into two $30^{\circ}$ partials $^{[44]}$ :

$$
\begin{gathered}
A=\left(\frac{1}{4}-\frac{1}{12(1-\nu)}\right) \frac{\mu b^{2}}{2 \pi} \\
F^{g}=\gamma_{i}-b \sigma_{E}^{g} /(2 \sqrt{3})
\end{gathered}
$$

The convention used in this framework is that positive $\sigma_{E}^{g}$ increases the distance between the partials and negative decreases it.

Analogously, if the dislocation does not move in the cross-slip plane, its equilibrium distance $l^{c s}$ is given by:

$$
l^{c s}=\frac{A}{F^{c s}}
$$

where

$$
F^{c s}=\gamma_{i}-b \sigma_{E}^{c s} /(2 \sqrt{3})
$$

The shape of the partials is described by continuous functions $y_{1}(x)$ and $y_{2}(x)$ (see Fig. 4). The constriction points are located in the $x$ axis at $\pm x_{d}$ and thus $y_{1}\left( \pm x_{d}\right)=y_{2}\left( \pm x_{d}\right)=0$ must be satisfied.

The energy contribution from the cross-slipped segment (measured with respect to the energy of an infinite straight screw dislocation resting at equilibrium in the glide plane) is given by ${ }^{[2]}$ :

$$
E^{c s}=W_{1}+W_{2}+W_{3}
$$

where $W_{1}$ is the change in elastic repulsion between the partials, $W_{2}$ is the change of the line energy and $W_{3}$ the change in SF energy and the work done by the stress.

Specifically,

$$
\begin{gathered}
W_{1}=A \int_{-x_{d}}^{x_{d}} \ln \left(\frac{l^{g}}{y_{1}-y_{2}}\right) d x \\
W_{2}=T \int_{-x_{d}}^{x_{d}}\left(\sqrt{1+\left(y_{1}^{\prime}\right)^{2}}+\sqrt{1+\left(y_{2}^{\prime}\right)^{2}}\right) d x-4 T x_{d} \\
W_{3}=\int_{-x_{d}}^{x_{d}}\left(F_{1}^{c s} y_{1}+F_{2}^{c s} y_{2}\right) d x-2 F^{g} l^{g} x_{d}
\end{gathered}
$$


where

$$
\begin{gathered}
F_{1}^{c s}=F^{c s}-b \sigma_{S}^{c s} / 2 \\
F_{2}^{c s}=-F^{c s}-b \sigma_{S}^{c s} / 2
\end{gathered}
$$

The energy functional in Eq. 26 was minimized using the conjugate gradient method as suggested by Kang et al. ${ }^{[2]}$.

The line energy per unit length was given by ${ }^{[2]}$ :

$$
T=\alpha \mu b^{2} / 2
$$

The variable $\alpha$ was treated as a fitting parameter to match the DD simulations of cross-slip (see section 3).

Formally, the energy barrier is given by:

$$
E_{b}\left(\sigma_{E}^{g}, \sigma_{S}^{c s}, \sigma_{E}^{c s}\right)=\max _{x_{d}} E\left(x_{d} ; \sigma_{E}^{g}, \sigma_{S}^{c s}, \sigma_{E}^{c s}\right)
$$

where

$$
E=\min _{y_{1}, y_{2}} E^{c s}\left(y_{1}, y_{2} ; x_{d} ; \sigma_{E}^{g}, \sigma_{S}^{c s}, \sigma_{E}^{c s}\right)+E^{g}\left(\sigma_{E}^{g}\right)
$$

$E^{g}$ being the energy of an isolated constriction on a perfect screw dislocation:

$$
E^{g}=2 l^{g} \sqrt{A T} \int_{0}^{1}[-\ln t+(t-1)]^{1 / 2} d t
$$

The activation energy can be obtained for a given stress condition by solving Eq. 34 numerically. Another useful formulation of the LT model of cross-slip was proposed by A. Malka-Markovitz et. al. ${ }^{[4]}$, who solved Strohs's LT model ${ }^{[23]}$ exactly by linearizing the interaction force between the partials. They obtained the following general expression for the activation enthalpy as function of the elastic constants and local-stress components:

$$
\begin{array}{r}
\frac{\Delta H(\sigma)}{\Delta E_{0}}=\frac{\beta^{g}}{2}+\left(\frac{\beta^{c s}}{2}\right) \tanh \left(l_{C}\right) \\
-\left(\frac{\beta^{c s}}{2}\right)\left[\frac{2 \alpha_{L}}{1.55} l_{C} E^{*}+\frac{\alpha_{L s}^{3}}{1.55} \frac{\delta}{\beta^{c s}} l_{C}^{3}\right]
\end{array}
$$

where $\Delta E_{0}=1.9 \mathrm{eV}$ is the energy barrier of copper obtained in this work using DD simulations and $\alpha_{L} \approx 0.6$ is a scale factor.

$\beta^{g}$ and $\beta^{c s}$ are functions of the Escaig stress: 


\begin{tabular}{|c|c|}
\hline Property & Value \\
\hline$a_{0}$ & {$[46] 3.61 \AA$} \\
$b$ & ${ }^{[47]} a_{0} / \sqrt{2}$ \\
$\gamma_{i}$ & ${ }^{[48]} 42 \mathrm{~mJ} / \mathrm{m}^{2}$ \\
$\gamma_{u}$ & ${ }^{[1]} 182 \mathrm{~mJ} / \mathrm{m}^{2}$ \\
$\nu$ & {$[49] 0.324$} \\
$\mu$ & {$[50] 54.6 \mathrm{GPa}$} \\
$B$ & ${ }^{[51]} 1.5 \times 10^{-5} \mathrm{~Pa}-\mathrm{S}$ \\
\hline
\end{tabular}

Table 1: Parameters used for FCC Cu; $a_{0}$ is the lattice parameter, $b$ the magnitude of the Burgers vector, $\gamma_{i}$ the intrinsic SF energy, $\gamma_{u}$ the unstable SF energy, $\nu$ the Poisson's ratio, $\mu$ the shear modulus and $B$ the viscosity coefficient at room temperature.

$$
\beta^{g}=\frac{1}{1+\frac{\sqrt{3} b}{6 \gamma_{i}} \sigma_{E}^{g}} \quad \text { and } \quad \beta^{c s}=\frac{1}{1+\frac{\sqrt{3} b}{6 \gamma_{i}} \sigma_{E}^{c s}}
$$

whereas $\delta$ is a quadratic function of the Schmid stress on the cross-slip plane:

$$
\delta=\frac{1}{6}\left(\frac{b \sigma_{S}^{c s}}{\gamma_{i}}\right)^{2}
$$

The $E^{*}$ function is a dimensionless off-set in the interaction energy between the glide and crossslip planes ${ }^{[45]}$ :

$$
E^{*}=\ln \left(\frac{\beta^{c s}}{\beta^{g}}\right)
$$

The critical length $l_{C}$ in Eq. 36 can be found by solving the following equation numerically ${ }^{[45]}$ :

$$
\frac{1.55}{\cosh ^{2}\left(l_{C}\right)}-\frac{3 \delta}{\beta^{c s}} l_{C}^{2}=2 E^{*}
$$

In contrast with the convention used in K. Kang's et al. ${ }^{[2]}$ LT model of cross-slip, positive $\sigma_{E}^{g}$ decreases the distance between the partials and negative increases it.

In this work, the sign convention of K. Kang's et al. ${ }^{[2]}$ was used in the plots.

The elastic constants of $\mathrm{Cu}$ used in both the DD simulations and LT model are shown in Table 1. 


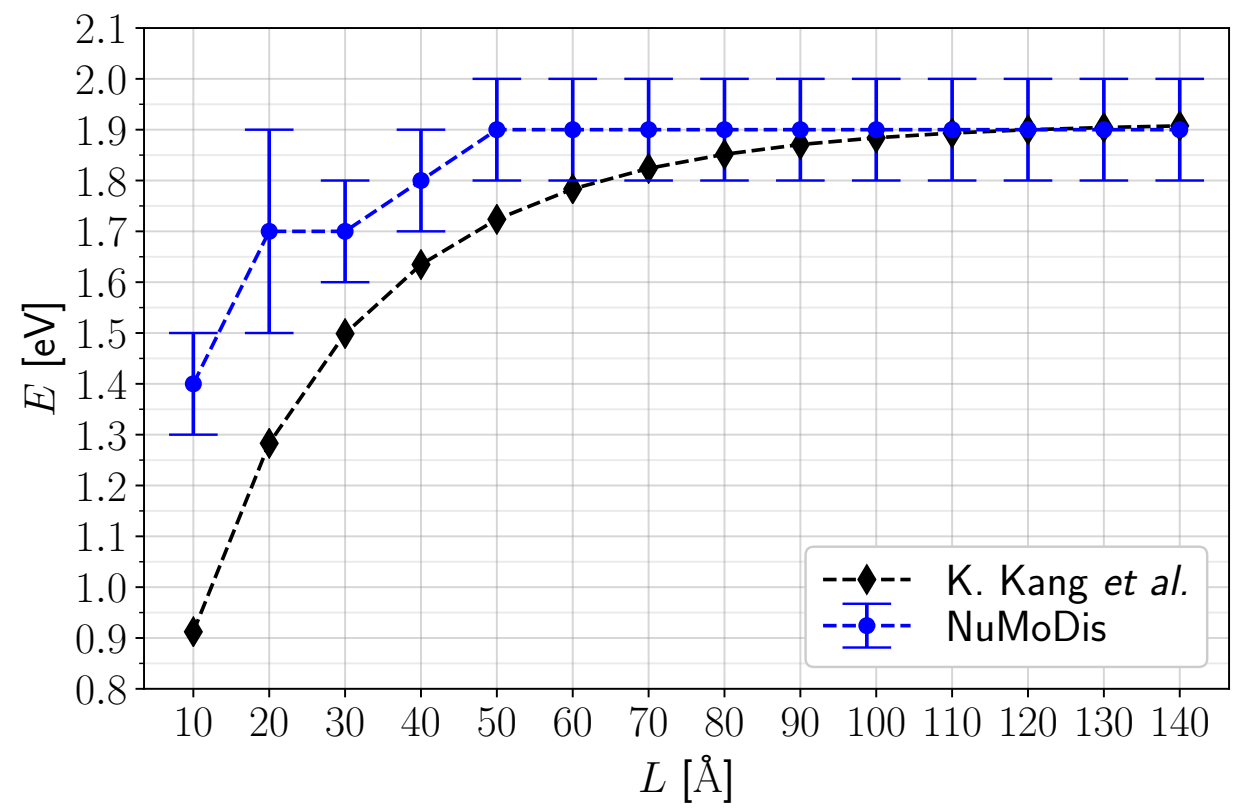

Figure 5: The energy barrier as function of the constriction separation at zero stress.

\section{Results and discussion}

\subsection{The calibration of the line tension model}

The relative energy of the intermediate cross-slip configurations at zero stress is shown in Fig. 5. The energy uncertainty of the order of $\pm 0.1 \mathrm{eV}$ in all the simulations results can be attributed to the line discretization used in the DD simulations, as well as to the finite core-width size of the nonsingular theory of dislocations. The parameter $\alpha$ in K. Kang's et al. ${ }^{[2]}$ LT model was calibrated such that the equilibration energy matched with the DD simulations at zero stress. The value was found to be $\alpha=0.22$. Together with the core-width parameter in the elastic energy, the choice of $\alpha$ was a key parameter for the good agreement between K. Kang's et al. ${ }^{[2]}$ LT model and DD simulations. The effect of compressive Escaig stress was to decrease the SF width. The stressed equilibrium separation obtained from the DD simulations was in great agreement with the LT model, as shown in Fig. 6.

The energy predicted by the K. Kang's et al. ${ }^{[2]}$ LT model and that obtained with the simulations does not match for short cross-slipped segments as seen in Fig. 5. This is expected because the LT model approximates the energy of a differential segment as that of a straight dislocation ${ }^{[52]}$. In fact, the LT model breaks down in the limiting case were $2 x_{d}=0$ because the elastic repulsion diverges (see Eq. 27). However, this is not relevant since the objective of this work is to quantify the stress effect on the energy barrier, which occurs for longer dislocation lines where the LT model is more physical. 


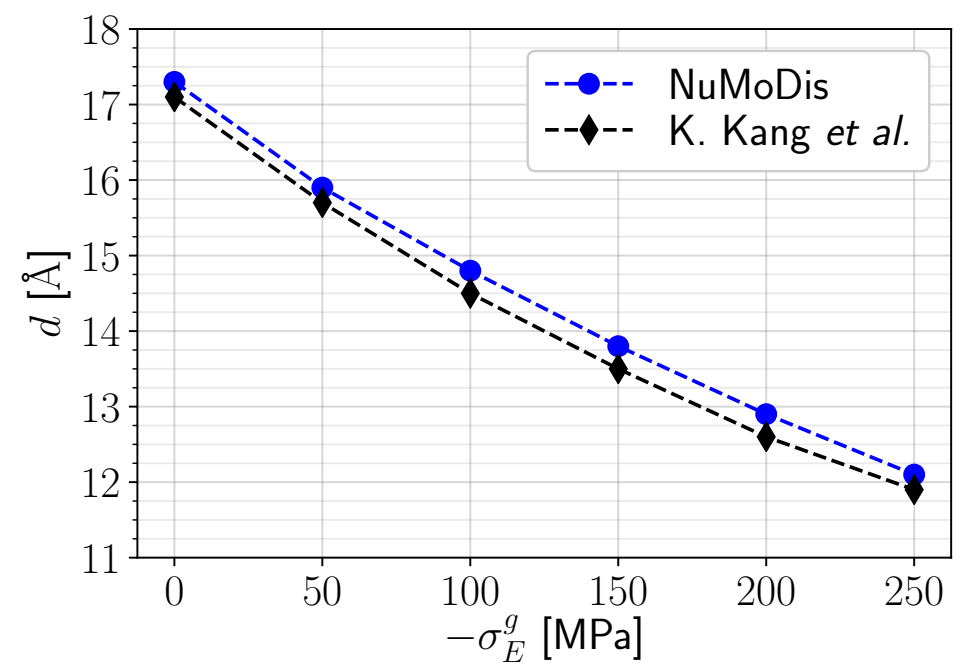

Figure 6: The effect of stress on the equilibrium separation between the partials. The segmented line corresponds to equation 21.

In the unstressed case, the energy monotonically augments as the constriction separation increases, but quickly reaches a maximum value of $1.9 \mathrm{eV}$. This means that once the cross-slip segment reaches a critical length (around $60 \AA$ ), no further energy is required to be invested and the cross-slip process continues spontaneously. Therefore, the unstressed energy barrier of copper is equal to $1.9 \mathrm{eV}$, as reported by B. Ramírez et al. ${ }^{[1]}$.

The equilibration energy encountered in the unstressed cross-slip is roughly equal to twice the energy required to form an isolated constriction. It can be argued that at the beginning of cross-slip, when the two constrictions are close to each other, the elastic repulsion between them adds up to the energy required to form them in isolation. However, as the distance between them increases, their interaction further decreases. Thus, if they are sufficiently far apart, the total energy with respect to the relaxed dislocation is roughly equal to that of the two independent constrictions. The planar constriction energy at zero stress is $0.7 \mathrm{eV}$, according to the DD simulations (see Fig. 7).

A. Stroh et al. ${ }^{[23]}$ found the energy of a constriction using elasticity theory. Assuming a shear modulus of $\mathrm{G}=45 \mathrm{GPa}$ and an equilibration distance between the partials at zero stress of $d_{0}=18$ $\AA$, one finds that in his model the energy of a single constriction is roughly $2 \mathrm{eV}$, which is almost three times higher than that found in this work $(0.7 \mathrm{eV})$.

W. Püschl ${ }^{[53]}$ estimated a constriction energy of $1.1 \mathrm{eV}$ for FCC $\mathrm{Cu}$ using elasticity theory; G. Saada ${ }^{[54]}$ generalized A. Strohs's LT model for arbitrary constricting distance. He obtained different constriction energy values in the range of $0.33-0.7 \mathrm{eV}$, depending on the choice $r_{0} / b$, where $r_{0}$ is a cut-off radius. Although these values are in better agreement with the experimental energy barrier, the (arbitrary) choice of $r_{0}$ was not clearly determined.

T. Rasmussen et al. ${ }^{[34]}$ found a constriction energy of $1.6 \mathrm{eV}$ using atomistic simulations, in 


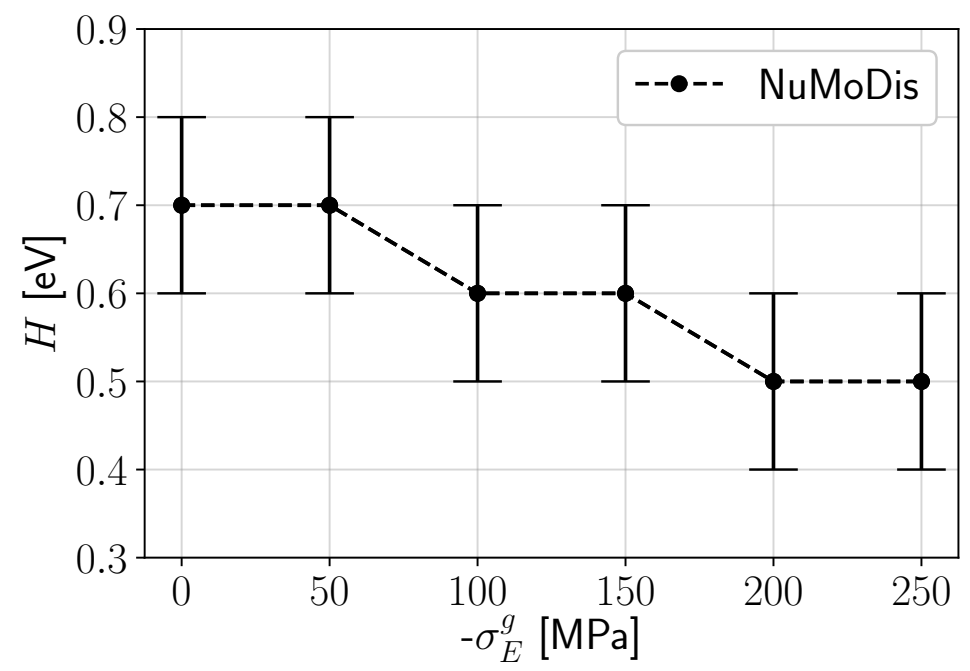

Figure 7: The effect of compressive stress on the constriction energy according to DD simulations.

reasonable agreement with A. Stroh et al. ${ }^{[23]}$, but too high according to the experimental energy barrier.

More recently, B. Ramírez et al. ${ }^{[1]}$ found a constriction energy of $0.7 \mathrm{eV}$ using dislocation dynamics, in excellent agreement with this work.

The effect of pure compressing stress on the constriction energy was found to be negligible. The slight decrease on the constriction energy observed in Fig. 7 is not conclusive. Nevertheless, one can assert that the average constriction energy did not vary much in the given range of stress.

The small effect of stress on the constriction energy can be explained by using the model developed by W. Püschl ${ }^{[53]}$. It predicts a marginal decrease in constriction energy (of less than $0.2 \mathrm{eV}$ ) when the splitting width between the partials decreases from $8 b \approx 20.4 \AA$ to $6 b \approx 15.3 \AA$. These are roughly the equilibration distances simulated by NuMoDis ${ }^{[38]}$ at zero $(17.4 \AA)$ and $-250 \mathrm{MPa}(12.1$ $\AA)$, respectively. Thus, according to this model, one should not expect the constriction energy to vary much by applying a pure compressing stress for the given range of values.

The asymptotic convergence of the cross-slip energy barrier at zero stress observed in Fig. 5 has been thoroughly reported before.

M. S. Duesbery et al ${ }^{[55]}$ calculated the constriction pair energy as function of the stackingfault energy using a linear-elastic framework, and found that for constriction separation larger than $\sim 50 b$, the total energy becomes that of two independent constrictions. In particular, for FCC $\mathrm{Cu}$, they found that the asymptotic value of the cross-slipped configuration was $3.7 \mathrm{eV}$. They also identified the asymmetric nature of the two constrictions in the cross-slip plane, as they asserted that one had lower energy being screw-like, and the other higher energy being edge-like. These are different from the Stroh-type constriction, which are edge-like on the one side, and screw-like on the other. 


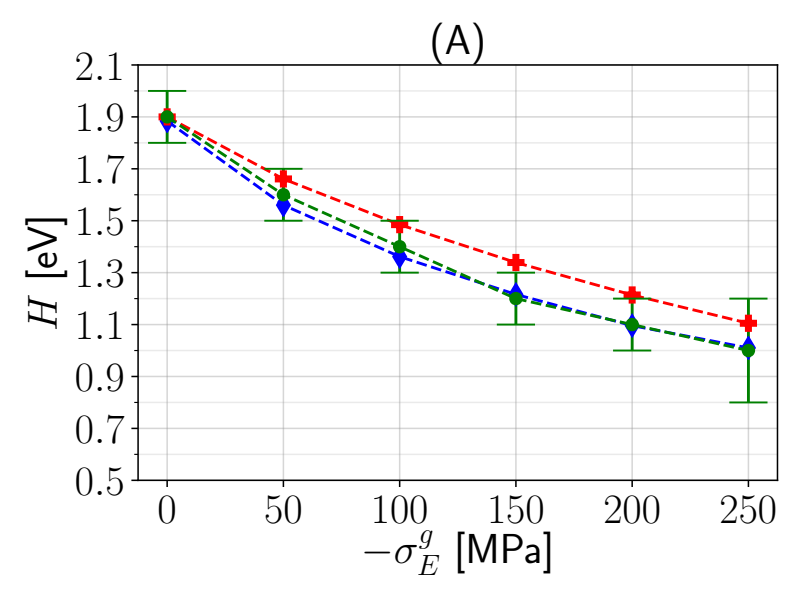

(B)

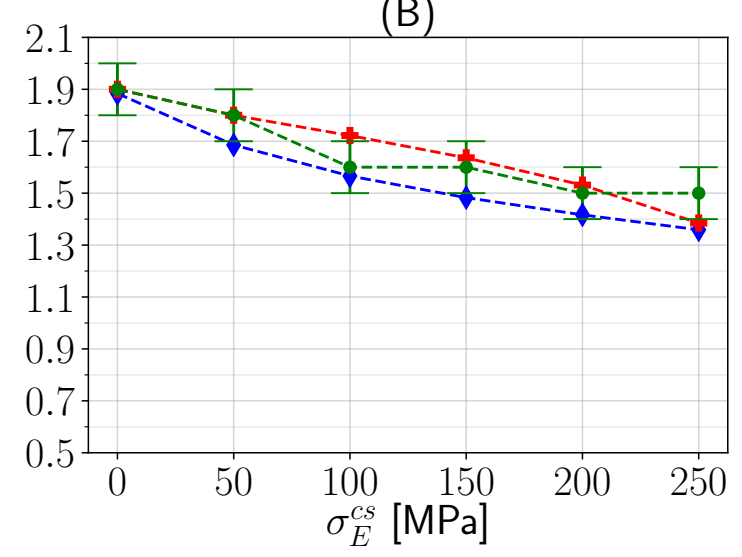

(C)

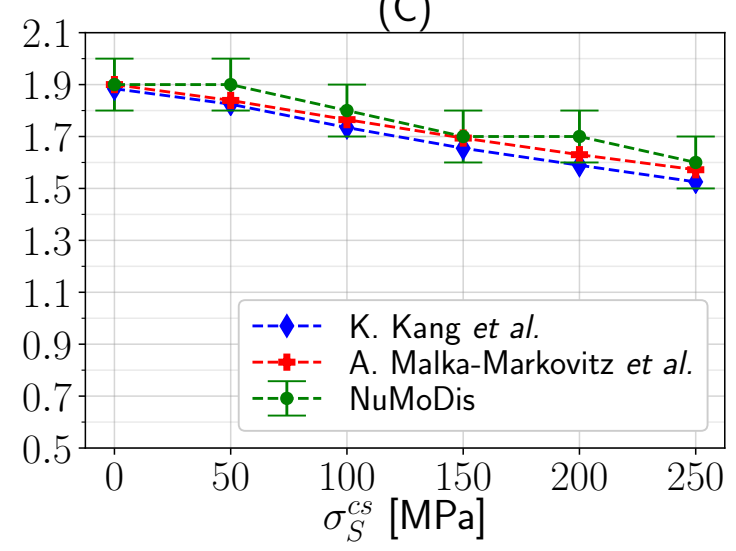

Figure 8: The effect of stress on the energy barrier: (A) $\sigma_{S}^{g}=\sigma_{E}^{c s}=\sigma_{S}^{c s}=0$, (B) $\sigma_{S}^{g}=\sigma_{E}^{g}=\sigma_{S}^{c s}=0$ and $(\mathrm{C}) \sigma_{S}^{g}=\sigma_{E}^{g}=\sigma_{E}^{c s}=0$. The green circles represent the DD simulation results, the blue diamonds correspond to the numerical solution of K. Kang's et al. ${ }^{[2]}$ LT model of cross-slip (see Eq. 34) and the red crosses were obtained from the general expression for the activation enthalpy proposed by A. Malka-Markovitz et. al. ${ }^{[4]}$ (see Eq. 36). 
Later on, T. Rasmussen et al. ${ }^{[34]}$ corroborated several results of M. S. Duesbery et al. ${ }^{[55]}$ using atomistic simulations, as they obtained that indeed the total energy converged to the value of two independent constrictions for separations larger than $\sim 50 b$. They measured a screw-like constriction energy of $-3.8 \mathrm{eV}$, and an edge-like constriction energy of $1.1 \mathrm{eV}$, which added up to the total energy barrier in FCC Cu $(2.7 \mathrm{eV})$. Although this value is lower than that obtained by M. S. Duesbery et $a l^{[55]}$, it is roughly the double of the experimental value obtained by J. Bonneville et al. ${ }^{[3]}$.

S. I. Rao et al. ${ }^{[30]}$ obtained an energy barrier for cross-slip at zero stress in the range of 1.07 - $1.28 \mathrm{eV}$ using molecular statics. This energy is significantly smaller than that predicted by $\mathrm{T}$. Rasmussen et al. ${ }^{[34]}$. They argued that it could be due to the difference in interatomic potentials used, as well as the Green's function technique used to relax the boundary forces in the simulations developed by S. I. Rao et al.

B. Ramírez et al. ${ }^{[1]}$ found an unstressed energy barrier of $1.9 \mathrm{eV}$ using DD simulations for dislocations dissociated into two partials. They obtained that the energy plateau was reached for a constriction separation of about $60 \AA$. Both results are in excellent agreement with the simulations in this work. Furthermore, notice that the critical length is about the half of the value found by previous methods.

The total energy of a solid crystal is all potential energy (assuming of course that the crystal does not move). The potential energy of an elastic system is the difference between the internal energy $\Pi^{\text {int }}$ and the energy due to external forces $\Pi^{\text {ext }[56]}$. The $\Pi^{\text {int }}$ consists of the stress-strain energy due to the stress field introduced by the dislocations (and their interactions) respect to the initial geometry i.e. the perfect crystal. The $\Pi^{\text {ext }}$ consists of the work done by the applied forces, which is obtained by multiplying the displacement generated by the dislocations as they move with the applied forces. Therefore, the energy barrier can be decreased in presence of an external stress.

In many DD fatigue simulations ${ }^{[10-14]}$, following the pioneering work of L. Kubin et al. ${ }^{[15]}$, the driving force for cross-slip is the Schmid stress. However, the screw dislocation does not really move because an obstacle is assumed to stop its motion in the glide plane. Thus, the effect of $\sigma_{S}^{g}$ is to compress the dislocation against the obstacle, reducing the equilibration distance between the partials ${ }^{[2]}$. In this way, the $\sigma_{S}^{g}$ stress plays a similar role to $\sigma_{E}^{g}$, if the dislocations is assumed to stop completely by being compressed against an obstacle. For this reason, $\sigma_{S}^{g}$ is assumed to be zero in all simulations, following the model developed by B. Escaig ${ }^{[21]}$.

\subsection{Uncoupled stress}

In order to study the independent influence of the stress components on the energy barrier, three cross-slip possibilities were considered: (A) pure compression on the glide plane, (B) pure expansion on the conjugate plane and $(\mathrm{C})$ pure bowing-out on the conjugate plane. The stress was increased up to $250 \mathrm{MPa}$, because the equilibrium configuration breaks down around $260 \mathrm{MPa}^{[57]}$.

The activation enthalpy obtained from DD simulations, the numerical solution of K. Kang's et al. ${ }^{[2]}$ LT model of cross-slip and the general expression for the activation enthalpy proposed by A. Malka-Markovitz et. al. ${ }^{[4]}$ are in good quantitative agreement with each other, as shown in Fig. 8. 
The largest influence on the energy barrier was obtained by pure compression on the glide plane, which reduced it up to $45 \%$ (see Fig. 8 (A)). The minimum energy barrier of $1 \mathrm{eV}$ was obtained by applying a compressive stress of $250 \mathrm{MPa}$ on the glide plane. This is in contrast with the lower energy value of $0.95 \mathrm{eV}$ obtained by $\mathrm{B}$. Ramírez et al. ${ }^{[1]}$ under a pure $\sigma_{E}^{c s}$ of $220 \mathrm{MPa}$. On the other hand, K. Kang et al. ${ }^{[2]}$ also concluded that the compressive stress applied on the glide was the most effective way of reducing the energy barrier in FCC nickel. The pure widening stress on the cross-slip reduced it up to $20 \%$ (see Fig. 8 (B)) and the pure bowing-out stress on the cross-slip produced a decrease in the energy barrier of about 15\% (see Fig. 8 (C)).

The effect of stress was not only to decrease the energy barrier but also the activation length of cross-slip. The maximum of energy at $200 \mathrm{MPa}$ occurred for a constriction separation of about 25 $\AA$. This value is comparable to the activation length of $12 b \approx 30.6 \AA$ at $220 \mathrm{MPa}$ obtained by B. Ramírez et al. ${ }^{[1]}$.

\subsection{Coupled stress}

In order to study the combined effect of stress on the energy barrier, three cross-slip possibilities were considered: (A) compression on the glide plane and expansion on the conjugate plane, (B) compression on the glide plane and bowing-out on the conjugate plane and (C) expansion and bowing-out on the conjugate plane.

As in the previous case, the activation enthalpy obtained from DD simulations and the two formulations of the LT model of cross-slip analyzed in this work ${ }^{[2,4]}$ are in good agreement (see Fig. 9).

The effect of applying a coupling compressive stress on the glide plane lead to the largest decrease in the energy barrier (see Figs. 9 (A) and (B)). These results resemble the effect applying an uncoupled compressive stress on the glide plane (see Fig. 8 (A)), which also lead to an energy barrier reduction of roughly $50 \%$.

The effect of applying coupled stresses on the conjugate plane only (see Fig. 9 (C)) was comparable of applying an uncoupled Schmid stress on the conjugate plane (see Fig. 8 (C)). In both cases, the energy barrier decreased up to $20 \%$.

These observations are validated by the atomistic simulations of G. Esteban-Manzanares et. $a l .{ }^{[58]}$, who studied the effect of stress in aluminum using molecular dynamics and obtained the same qualitative results.

\section{Summary and Conclusions}

In this work, the unstressed constriction energy was equal to $0.7 \mathrm{eV}$, as reported by B. Ramírez et al. ${ }^{[1]}$. The effect of pure compressive stress in the range of -50 to $-250 \mathrm{MPa}$ on the constriction energy was negligible, in agreement with W. Püschl ${ }^{[53]}$.

The unstressed energy barrier was found to be $1.9 \mathrm{eV}$, as obtained by B. Ramírez et al. ${ }^{[1]}$. However, it does not lie within the experimental range of $1.15 \pm 0.37 \mathrm{eV}$ measured by J. Bonneville et al. ${ }^{[3]}$. On the other hand, the energy barrier could be reduced by applying an external stress, 

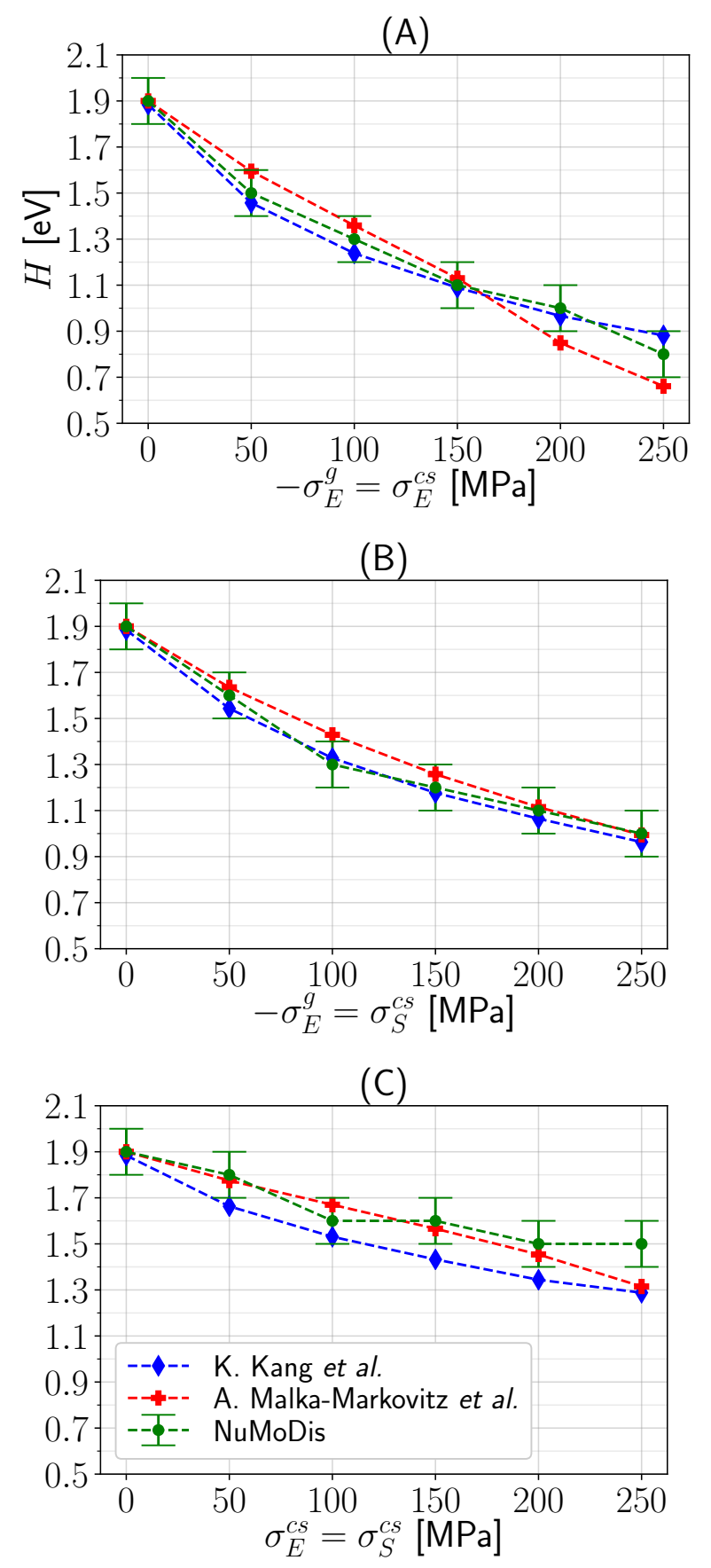

Figure 9: The effect of stress on the energy barrier: (A) $\sigma_{S}^{g}=\sigma_{S}^{c s}=0,(\mathrm{~B}) \sigma_{S}^{g}=\sigma_{E}^{c s}=0$ and $(\mathrm{C})$ $\sigma_{S}^{g}=\sigma_{E}^{g}=0$. 
leading to an energy barrier in better agreement with the experimental results. The most effective way of reducing it, was by pure compression on the glide plane. Furthermore, it was found using DD simulations, that both the Schmid and Escaig stress on the conjugate plane have a comparable effect in reducing the energy barrier, in qualitative agreement with the atomistic simulations performed by K. Kang et al. ${ }^{[2]}$ in FCC nickel.

The energy barrier of FCC $\mathrm{Cu}$ at zero stress has been obtained using the line LT model and atomistic simulations before. Some of them reported an energy barrier in better agreement with experiments ${ }^{[30,54]}$. However, the LT model suffers from arbitrariness in the cut-off radius determination $^{[54]}$ and the atomistic simulations are highly sensitive to the interatomic potential used ${ }^{[30]}$. In DD simulations, these problems do not exist and the core-width used to compute the elastic energy can be calculated using the theory developed by G. Schöck ${ }^{[35]}$ as suggested by B. Ramírez et $a l .{ }^{[1]}$. Moreover, the effect of non-homogeneous stress on the energy barrier, like those generated by pile-ups and forest dislocations, can be more naturally studied using DD simulations, in comparison with other simulation techniques.

The activation enthalpy obtained from DD simulations was in excellent agreement with both the numerical solution of K. Kang's et al. ${ }^{[2]}$ LT model of cross-slip and the general expression for the activation enthalpy proposed by A. Malka-Markovitz et. al. ${ }^{[4]}$. Since the later is an analytical model, it can be easily implemented in a DD code to calculate the activation enthalpy of screw segments.

Acknowledging a limitation of this work, a perfect screw dislocation in FCC crystals can be dissociated in a set of fractional partial dislocations (FPD) pairs, instead of two Shockley partials with Burgers vectors $\boldsymbol{b}_{\mathbf{1}}$ and $\boldsymbol{b}_{\mathbf{2}}$. Based on the work of S. Banerjee et al. ${ }^{[59]}$, B. R. Ramírez et al. ${ }^{[1]}$ generalized the dissociation of perfect screw dislocations into $n$ pairs of fractional partial dislocations (FPD) with Burgers vectors $\boldsymbol{b}_{\mathbf{1}} / \mathrm{n}$ and $\boldsymbol{b}_{\mathbf{2}} / \mathrm{n}$. By using 20 FPDs, they obtained an energy barrier reduction from 1.9 to $1.43 \mathrm{eV}$ at zero stress, which lies within the experimental error of the energy barrier measured by J. Bonneville et al. ${ }^{[3]}$.

Regarding the LT model, the constant line energy per unit length $T$ makes it impossible to distinguish between edge-like and screw-like constrictions. Thus, a more physical description of cross-slip can be made by letting the total energy depend on the angle between the Burgers vector and the local tangent vector ${ }^{[2,45,52]}$. In spite of it, the energy barrier calculated using the LT model was found to be in good quantitative agreement with the DD simulations, at least for the case of dissociation into two partials. Hence, the orientation-dependent LT model might be in better agreement with DD simulations that describe the dislocation core using more than a pair of FPDs.

\section{Acknowledgments}

The authors acknowledge use of the 3D dislocation dynamics code NuMoDis jointly developed at CEA (L. Dupuy) and Univ. Paris XII (M. Bletry). This work has received funding from the European Research Council under the European Union's ERC grant agreement number 725142. 


\section{References}

[1] B. Ramírez, N. Ghoniem, G. Po, Ab initio continuum model for the influence of local stress on cross-slip of screw dislocations in fcc metals, Physical Review B 86 (9) (2012) 1-11. doi: 10.1103/PhysRevB.86.094115.

URL https://link.aps.org/doi/10.1103/PhysRevB.86.094115

[2] K. Kang, J. Yin, W. Cai, Stress dependence of cross slip energy barrier for face-centered cubic nickel, Journal of the Mechanics and Physics of Solids 62 (2014) 181-193. doi:10.1016/j. jmps.2013.09.023.

URL https://linkinghub.elsevier.com/retrieve/pii/S002250961300207X

[3] J. Bonneville, B. Escaig, J. Martin, A study of cross-slip activation parameters in pure copper, Acta Metallurgica 36 (8) (1988) 1989-2002. doi:10.1016/0001-6160(88)90301-X. URL https://linkinghub.elsevier.com/retrieve/pii/000161608890301X

[4] A. Malka-Markovitz, D. Mordehai, Cross-slip in face centred cubic metals: a general full stressfield dependent activation energy line-tension model, Philosophical Magazine 99 (12) (2019) 1460-1480. doi:10.1080/14786435.2019.1584410.

URL https://www.tandfonline.com/doi/full/10.1080/14786435.2019.1584410

[5] P. Anderson, J. Hirth, J. Lothe, Theory of Dislocations, Cambridge University Press, 2017.

URL https://books.google.fr/books?id=LK7DDQAAQBAJ

[6] P. Jackson, The role of cross-slip in the plastic deformation of crystals, Materials Science and Engineering 57 (1) (1983) 39-47. doi : 10.1016/0025-5416(83)90025-3.

URL https://linkinghub.elsevier.com/retrieve/pii/0025541683900253

[7] P. Hirsch, The Physics of Metals, Cambridge University Press, 2009.

URL https://books.google.fr/books?id=5JOKPwAACAAJ

[8] P. Jackson, Dislocation modelling of shear in f.c.c. crystals, Progress in Materials Science 29 (12) (1985) 139-175. doi:10.1016/0079-6425(85)90009-X.

URL https://linkinghub.elsevier.com/retrieve/pii/007964258590009X

[9] M. Sudmanns, M. Stricker, D. Weygand, T. Hochrainer, K. Schulz, Dislocation multiplication by cross-slip and glissile reaction in a dislocation based continuum formulation of crystal plasticity, Journal of the Mechanics and Physics of Solids 132 (2019) 1-19. doi: $10.1016 / j \cdot j m p s .2019 .103695$.

URL https://linkinghub.elsevier.com/retrieve/pii/S0022509619306428

[10] H. Zbib, M. Rhee, J. Hirth, On plastic deformation and the dynamics of 3D dislocations, International Journal of Mechanical Sciences 40 (2-3) (1998) 113-127. doi:10.1016/ 
S0020-7403(97) 00043-X.

URL https://linkinghub.elsevier.com/retrieve/pii/S002074039700043X

[11] D. Weygand, L. Friedman, E. Giessen, A. Needleman, Aspects of boundary-value problem solutions with three-dimensional dislocation dynamics, Modelling and Simulation in Materials Science and Engineering 10 (4) (2002) 437-468. doi:10.1088/0965-0393/10/4/306. URL https://iopscience.iop.org/article/10.1088/0965-0393/10/4/306

[12] M. Verdier, M. Fivel, I. Groma, Mesoscopic scale simulation of dislocation dynamics in fcc metals: Principles and applications, Modelling and Simulation in Materials Science and Engineering 6 (1998) 755-770. doi:10.1088/0965-0393/6/6/007.

URL https://iopscience.iop.org/article/10.1088/0965-0393/6/6/007

[13] C. Déprés, C. Robertson, M. Fivel, 3D Discrete Dislocation Dynamics Investigations of Fatigue Crack Initiation and Propagation, Aerospace Lab Journal AL09-01 (9) (2015) 1-8. doi:10. 12762/2015. AL09-01.

[14] J. Chaussidon, C. Robertson, D. Rodney, M. Fivel, Dislocation dynamics simulations of plasticity in Fe laths at low temperature, Acta Materialia 56 (19) (2008) 5466-5476. doi: $10.1016 / j$.actamat. 2008.07.047.

URL http://dx.doi.org/10.1016/j.actamat.2008.07.047

[15] L. Kubin, G. Canova, M. Condat, B. Devincre, V. Pontikis, Y. Bréchet, Dislocation Microstructures and Plastic Flow: A 3D Simulation, Solid State Phenomena 23-24 (1992) 455-472. doi:10.4028/www.scientific.net/SSP.23-24.455.

URL https://www.scientific.net/SSP.23-24.455

[16] C. Déprés, C. Robertson, M. Fivel, Crack initiation in fatigue: experiments and threedimensional dislocation simulations, Materials Science and Engineering: A 387-389 (2004) 288-291. doi:10.1016/j.msea.2003.12.084.

URL https://linkinghub.elsevier.com/retrieve/pii/S0921509304007075

[17] B. Devincre, T. Hoc, L. Kubin, Collinear interactions of dislocations and slip systems, Materials Science and Engineering: A 400-401 (2005) 182-185. doi:10.1016/j.msea.2005.02.071. URL https://linkinghub.elsevier.com/retrieve/pii/S0921509305003333

[18] T. Nogaret, D. Rodney, M. Fivel, C. Robertson, Clear band formation simulated by dislocation dynamics: Role of helical turns and pile-ups, Journal of Nuclear Materials 380 (1-3) (2008) 22-29. doi:10.1016/j.jnucmat.2008.07.001.

URL https://linkinghub.elsevier.com/retrieve/pii/S002231150800367X 
[19] A. Hussein, S. Rao, M. Uchic, D. Dimiduk, J. El-Awady, Microstructurally based cross-slip mechanisms and their effects on dislocation microstructure evolution in fcc crystals, Acta Materialia 85 (2015) 180-190. doi:10.1016/j.actamat.2014.10.067.

URL http://dx.doi.org/10.1016/j.actamat.2014.10.067

[20] R. Fleischer, Cross slip of extended dislocations, Acta Metallurgica 7 (2) (1959) 134-135. doi:10.1016/0001-6160(59)90122-1.

URL https://linkinghub.elsevier.com/retrieve/pii/0001616059901221

[21] B. Escaig, Sur le glissement dévié des dislocations dans la structure cubique à faces centrées, Journal de Physique 29 (2-3) (1968) 225-239. doi:10.1051/jphys:01968002902-3022500. URL http://www.edpsciences.org/10.1051/jphys:01968002902-3022500

[22] J. Friedel, Dislocations: International Series of Monographs on Solid State Physics, Elsevier Science, 2013.

[23] A. Stroh, Constrictions and Jogs in Extended Dislocations, Proceedings of the Physical Society. Section B 67 (5) (1954) 427-436. doi:10.1088/0370-1301/67/5/307.

URL https://iopscience.iop.org/article/10.1088/0370-1301/67/5/307

[24] W. Püschl, Models for dislocation cross-slip in close-packed crystal structures: a critical review, Progress in Materials Science 47 (4) (2002) 415-461. doi:10.1016/S0079-6425(01)00003-2. URL https://linkinghub.elsevier.com/retrieve/pii/S0079642501000032

[25] C. Jin, Y. Xiang, G. Lu, Dislocation cross-slip mechanisms in aluminum, Philosophical Magazine 91 (32) (2011) 4109-4125. doi:10.1080/14786435.2011.602030.

URL http://www.tandfonline.com/doi/abs/10.1080/14786435.2011.602030

[26] S. Xu, L. Xiong, Y. Chen, D. McDowell, Shear stress- and line length-dependent screw dislocation cross-slip in FCC Ni, Acta Materialia 122 (2017) 412-419. doi:10.1016/j . actamat. 2016.10 .005$.

URL https://linkinghub.elsevier.com/retrieve/pii/S1359645416307601

[27] W. Kuykendall, Y. Wang, W. Cai, Stress effects on the energy barrier and mechanisms of cross-slip in FCC nickel, Journal of the Mechanics and Physics of Solids 144 (2020) 1-13. doi:10.1016/j.jmps.2020.104105.

URL https://doi.org/10.1016/j.jmps.2020.104105

[28] T. Rasmussen, K. Jacobsen, T. Leffers, O. Pedersen, S. Srinivasan, H. Jónsson, Atomistic Determination of Cross-Slip Pathway and Energetics, Physical Review Letters 79 (19) (1997) 3676-3679. doi:10.1103/PhysRevLett.79.3676.

URL https://link.aps.org/doi/10.1103/PhysRevLett.79.3676 
[29] T. Vegge, T. Rasmussen, T. Leffers, O. Pedersen, K. Jacobsen, Atomistic simulations of crossslip of jogged screw dislocations in copper, Philosophical Magazine Letters 81 (3) (2001) 137 144. doi:10.1080/09500830010019040.

URL http://www.tandfonline.com/doi/abs/10.1080/09500830010019040

[30] S. Rao, T. Parthasarathy, C. Woodward, Atomistic simulation of cross-slip processes in model fcc structures, Philosophical Magazine A 79 (5) (1999) 1167-1192. doi:10.1080/ 01418619908210354.

URL http://www.tandfonline.com/doi/abs/10.1080/01418619908210354

[31] S. Rao, D. Dimiduk, J. El-Awady, T. Parthasarathy, M. Uchic, C. Woodward, Atomistic simulations of cross-slip nucleation at screw dislocation intersections in face-centered cubic nickel, Philosophical Magazine 89 (34-36) (2009) 3351-3369. doi:10.1080/14786430903286201.

URL http://www.tandfonline.com/doi/abs/10.1080/14786430903286201

[32] S. Rao, D. Dimiduk, C. Woodward, T. Parthasarathy, On the Escaig obstacle hypothesis for cross-slip in face-centered-cubic materials, Philosophical Magazine Letters 91 (7) (2011) 452457. doi:10.1080/09500839.2011.581703.

URL https://www.tandfonline.com/doi/full/10.1080/09500839.2011.581703

[33] S. Rao, D. Dimiduk, J. El-Awady, T. Parthasarathy, M. Uchic, C. Woodward, Screw dislocation cross slip at cross-slip plane jogs and screw dipole annihilation in FCC $\mathrm{Cu}$ and Ni investigated via atomistic simulations, Acta Materialia 101 (2015) 10-15. doi:10.1016/j.actamat.2015. 08.070.

URL http://dx.doi.org/10.1016/j.actamat.2015.08.070

[34] T. Rasmussen, K. Jacobsen, T. Leffers, O. Pedersen, Simulations of the atomic structure, energetics, and cross slip of screw dislocations in copper, Physical Review B 56 (6) (1997) 2977-2990. doi:10.1103/PhysRevB.56.2977.

URL https://link.aps.org/doi/10.1103/PhysRevB.56.2977

[35] G. Schöck, Atomic dislocation core parameters, physica status solidi (b) 247 (2) (2010) 265268. doi:10.1002/pssb. 200945379.

URL http://doi.wiley.com/10.1002/pssb. 200945379

[36] R. Sills, W. Kuykendall, A. Aghaei, W. Cai, Fundamentals of Dislocation Dynamics Simulations, Springer International Publishing, 2016, pp. 53-87. doi :10.1007/978-3-319-33480-6_ 2.

URL https://doi.org/10.1007/978-3-319-33480-6\{_\}2

[37] The ParaDiS program, http://paradis.stanford.edu. 
[38] Numerical Modelling of Dislocations (NuMoDis).

URL http://www. numodis.fr/

[39] A. Arsenlis, W. Cai, M. Tang, M. Rhee, T. Oppelstrup, G. Hommes, T. Pierce, V. Bulatov, Enabling strain hardening simulations with dislocation dynamics, Modelling and Simulation in Materials Science and Engineering 15 (6) (2007) 553-595. doi : 10.1088/0965-0393/15/6/001. URL https://iopscience.iop.org/article/10.1088/0965-0393/15/6/001

[40] E. Martínez, J. Marian, A. Arsenlis, M. Victoria, J. Perlado, Atomistically informed dislocation dynamics in fcc crystals, Journal of the Mechanics and Physics of Solids 56 (3) (2008) 869-895. doi:10.1016/j.jmps.2007.06.014.

URL https://linkinghub.elsevier.com/retrieve/pii/S0022509607001408

[41] V. Bulatov, W. Cai, Computer Simulations of Dislocations, OUP Oxford, 2006. URL https://books.google.fr/books?id=AdfbPfihVJsC

[42] W. Cai, A. Arsenlis, C. Weinberger, V. Bulatov, A non-singular continuum theory of dislocations, Journal of the Mechanics and Physics of Solids 54 (3) (2006) 561-587. doi: $10.1016 / j \cdot j m p s .2005 .09 .005$.

URL https://linkinghub.elsevier.com/retrieve/pii/S002250960500195X

[43] G. Schoeck, The Peierls model: Progress and limitations, Materials Science and Engineering A 400-401 (1-2 SUPPL.) (2005) 7-17. doi:10.1016/j.msea.2005.03.050.

[44] W. Cai, V. Bulatov, J. Justo, A. Argon, S. Yip, Intrinsic Mobility of a Dissociated Dislocation in Silicon, Physical Review Letters 84 (15) (2000) 3346-3349. doi:10.1103/PhysRevLett. 84. 3346.

URL https://link.aps.org/doi/10.1103/PhysRevLett.84.3346

[45] A. Malka-Markovitz, D. Mordehai, Cross-slip in face-centered cubic metals: a general Escaig stress-dependent activation energy line tension model, Philosophical Magazine 98 (5) (2018) 347-370. doi:10.1080/14786435.2017.1406194.

URL https://doi.org/10.1080/14786435.2017.1406194

[46] W. Davey, Precision Measurements of the Lattice Constants of Twelve Common Metals, Physical Review 25 (6) (1925) 753-761. doi:10.1103/PhysRev.25.753.

URL https://link.aps.org/doi/10.1103/PhysRev.25.753

[47] C. Kittel, P. McEuen, Introduction to Solid State Physics, John Wiley \& Sons, 2018. URL https://books.google.fr/books?id=S3gAugEACAAJ

[48] J. Bonneville, B. Escaig, Cross-slipping process and the stress-orientation dependence in pure copper, Acta Metallurgica 27 (9) (1979) 1477-1486. doi:10.1016/0001-6160 (79)90170-6. URL https://linkinghub.elsevier.com/retrieve/pii/0001616079901706 
[49] H. Ledbetter, E. Naimon, Elastic Properties of Metals and Alloys. II. Copper, Journal of Physical and Chemical Reference Data 3 (4) (1974) 897-935. doi:10.1063/1.3253150. URL http://aip.scitation.org/doi/10.1063/1.3253150

[50] S. Schmauder, L. Mishnaevsky, Micromechanics and Nanosimulation of Metals and Composites: Advanced Methods and Theoretical Concepts, Springer Berlin Heidelberg, 2008. URL https://books.google.fr/books?id=QcyxI-3EG4cC

[51] J. Philibert, Dislocations et déformation plastique, Les éditions de la physique in École d'été d'Yravals 101 (1979).

[52] L. Dupuy, M. Fivel, A study of dislocation junctions in FCC metals by an orientation dependent line tension model, Acta Materialia 50 (19) (2002) 4873-4885. doi:10.1016/S1359-6454(02) 00356-7.

URL https://linkinghub.elsevier.com/retrieve/pii/S1359645402003567

[53] W. Püschl, The Energy of Constrictions in Extended Dislocations, physica status solidi (b) 162 (2) (1990) 363-370. doi:10.1002/pssb. 2221620205.

URL http://doi.wiley.com/10.1002/pssb. 2221620205

[54] G. Saada, Cross-slip and work hardening of f.c.c. crystals, Materials Science and Engineering: A 137 (1991) 177-183. doi:10.1016/0921-5093(91)90333-I.

URL https://linkinghub.elsevier.com/retrieve/pii/092150939190333I

[55] M. Duesbery, N. Louat, K. Sadananda, The mechanics and energetics of cross-slip, Acta Metallurgica et Materialia 40 (1) (1992) 149-158. doi:10.1016/0956-7151(92)90208-V. URL https://linkinghub.elsevier.com/retrieve/pii/095671519290208V

[56] N. Kim, Introduction to Nonlinear Finite Element Analysis, Springer US, 2014. URL https://books.google.fr/books?id=PDeSBQAAQBAJ

[57] A. Davis, M. Shokouhian, S. Ni, Loading estimates of lead, copper, cadmium, and zinc in urban runoff from specific sources, Chemosphere 44 (5) (2001) 997-1009. doi:10.1016/ S0045-6535(00)00561-0.

URL https://linkinghub.elsevier.com/retrieve/pii/S0045653500005610

[58] G. Esteban-Manzanares, R. Santos-Güemes, I. Papadimitriou, E. Martínez, J. LLorca, Influence of the stress state on the cross-slip free energy barrier in Al: An atomistic investigation, Acta Materialia 184 (2020) 109-119. arXiv:1910.14556, doi:10.1016/j.actamat.2019.10. 055.

URL https://doi.org/10.1016/j.actamat.2019.10.055 
[59] S. Banerjee, N. Ghoniem, G. Lu, N. Kioussis, Non-singular descriptions of dislocation cores: a hybrid ab initio continuum approach, Philosophical Magazine 87 (27) (2007) 4131-4150. doi:10.1080/14786430701528739.

URL http://www.tandfonline.com/doi/abs/10.1080/14786430701528739 

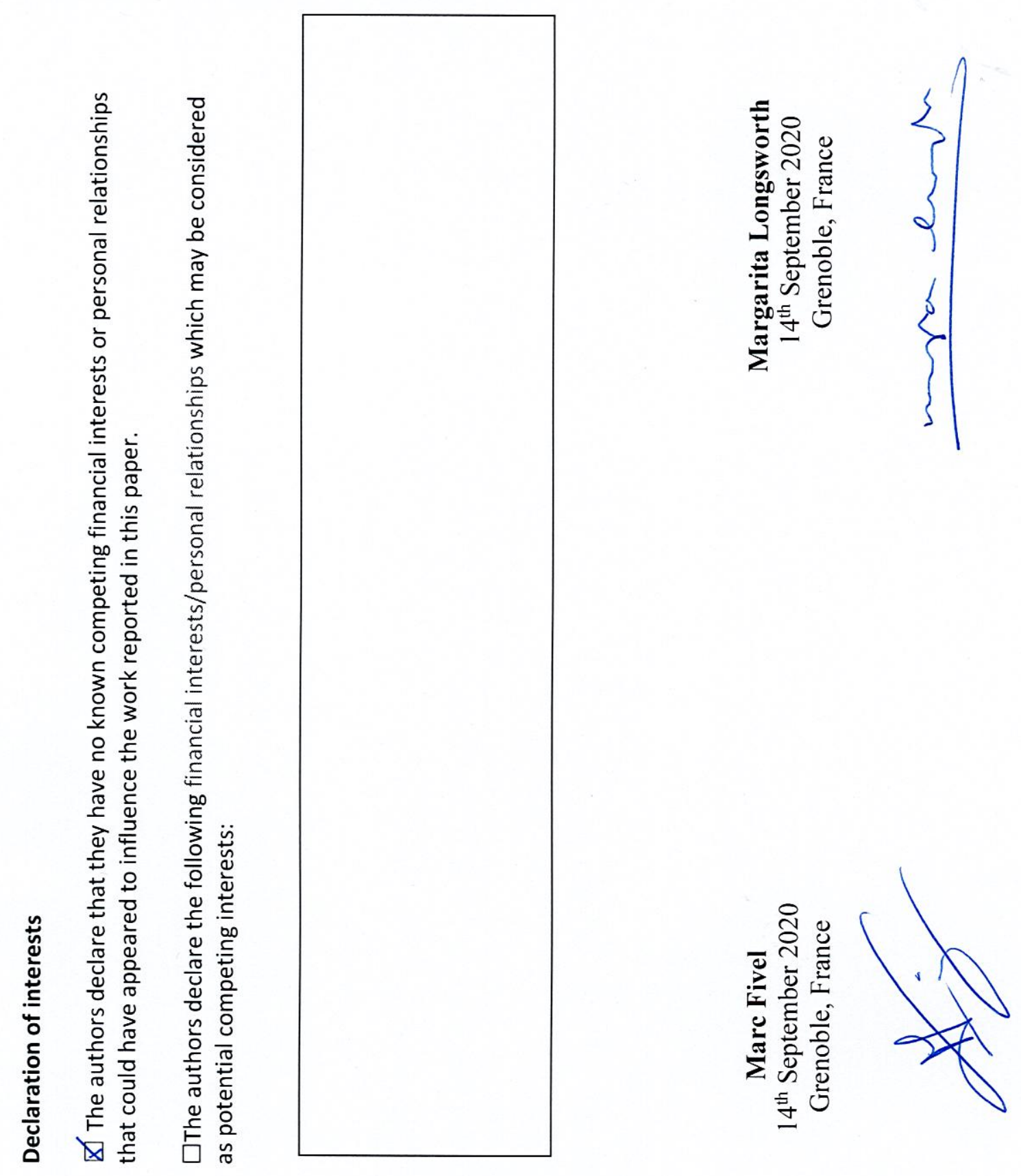
Margarita Longsworth: Conceptualization, Methodology, Software, Writing - original draft. Marc Fivel: Conceptualization, Supervision, Writing - review \& editing. 